\title{
Correction Of X-ray Images To Remove the Effect Of Asymmetric Source Shape
}

\author{
Peter Miller
}

XRT Limited, 86 Peters Avenue, Mulgrave, Victoria 3170, Australia.

pmiller@xrt.com.au.www.xrt.com.au

The projection method for X-ray microscopy is very simple in principle, see Figure 1. X-rays from the point-like source pass through the sample to form a projected image at the detector. Moving the sample position between the source and the detector varies magnification. The projection X-ray microscope is capable of providing high magnification over a wide range of $\mathrm{X}$-ray energies.

Point projection X-ray microscopy was first used in the early 1930s. Resolution of the point projection X-ray microscope is limited in part by the size of the X-ray source. Performance was improved in the late 1950s when magnetic lenses were used to focus an electron beam to provide a smaller X-ray source, see Cosslett and Nixon 1960 [1]. In 1978 Horn and Waltinger developed an $\mathrm{X}$-ray microscope using a scanning electron microscope to produce an even finer X-ray source [2]. However, the low current density of electron sources at that time resulted in low X-ray intensities and this combined with poor detection efficiency meant that very long exposure times were needed.

The subsequent development of high-brightness field-emission guns, high efficiency CCD X-ray detectors, new phase retrieval algorithms, automation of SEM operation and the ready availability of powerful desktop computers has allowed the development of a very much more capable laboratorybased X-ray microscope [3]. The X-ray ultraMicroscope (XuM) is an SEM-based X-ray microscope that allows X-ray images to be recorded with resolution better than $100 \mathrm{~nm}$. The system is highly automated and is capable of unattended operation. X-ray images from the XuM exploit both absorption and phase contrast to reveal fine internal structure and edge detail.

In comparison with existing microscopy techniques, the XuM offers:

- Ability to form images showing internal sample structure.

- Enhanced image detail using both absorption and phase contrast data.

- Large depth of field, allowing micro-tomography and stereo imaging.

The resolution of the XuM is in part determined by the X-ray source size. The XuM uses a thin foil target held normal to the electron beam to provide a sub-micron X-ray source. The X-ray detector views the foil edge on so that the projected volume of X-ray generation is strongly asymmetric in shape. A Monte Carlo program has been written to model the projected point spread function (PSF) for characteristic X-rays and for bremsstrahlung X-rays adapted from the methods given by Joy [4]. A calculated PSF for Ta L shell ionizations in a $500 \mathrm{~nm}$ foil at $30 \mathrm{kV}$ assuming a $50 \mathrm{~nm}$ Gaussian electron probe is shown in Figure 2c. Figure 2a shows an X-ray image as collected and Figure $2 b$ shows the same image after deconvolution to remove the effect of the source asymmetry [5]. In the original image the bright Fresnel fringe can be seen on three sides of the square features but not on the top side. In the deconvoluted image the Fresnel fringe has been restored at the top. Deconvoluted images are subsequently processed using phase retrieval methods to obtain a projected density image. The removal of the asymmetry in images has been found to simplify image interpretation. While in principle the PSF is energy dependent and should include both characteristic and 
bremsstrahlung X-rays, in practice good results have been obtained using only the characteristic Xray PSF. The X-ray detector is most sensitive in the range $4-12 \mathrm{keV}$; deconvolution of the PSF would be more difficult if the detector was operated in a windowless mode giving a wider energy range where more than one series of characteristic lines may be detected.

Measurements giving the distribution of X-ray generation within a sample are needed to validate models for X-ray generation. With suitable target, X-ray filter and sample it is possible to obtain information on the projected volume of X-ray generation within the target. The distribution of the focused electron beam must also be known. Using a circular object or hole a few microns in diameter as a sample the PSF can be estimated by determining the distribution of the X-ray source needed to transform the theoretical image of the circular feature into the experimental image. Alternatively the PSF could be estimated by examining the variation in the power spectrum with orientation of an image of randomly oriented irregularly shaped objects such as coarse gold islands.

\section{References}

[1] V.E. Cosslett \& W.C. Nixon (1960) X-ray Microscopy, Cambridge University Press, London [2] H.R.F. Horn \& H.G. Waltinger (1978), Scanning, 1, 100-108

[3] S.C. Mayo et al., Journal of Microscopy, Vol. 207, Pt 2 Aug. 2002, 79-96

[4] D.C. Joy (1995), Monte Carlo Modeling for Electron Microscopy and Microanalysis, Oxford University Press, New York.

[5] The aid of Dr S. Mayo and Dr T. Gureyev of CSIRO MIT is gratefully acknowledged.

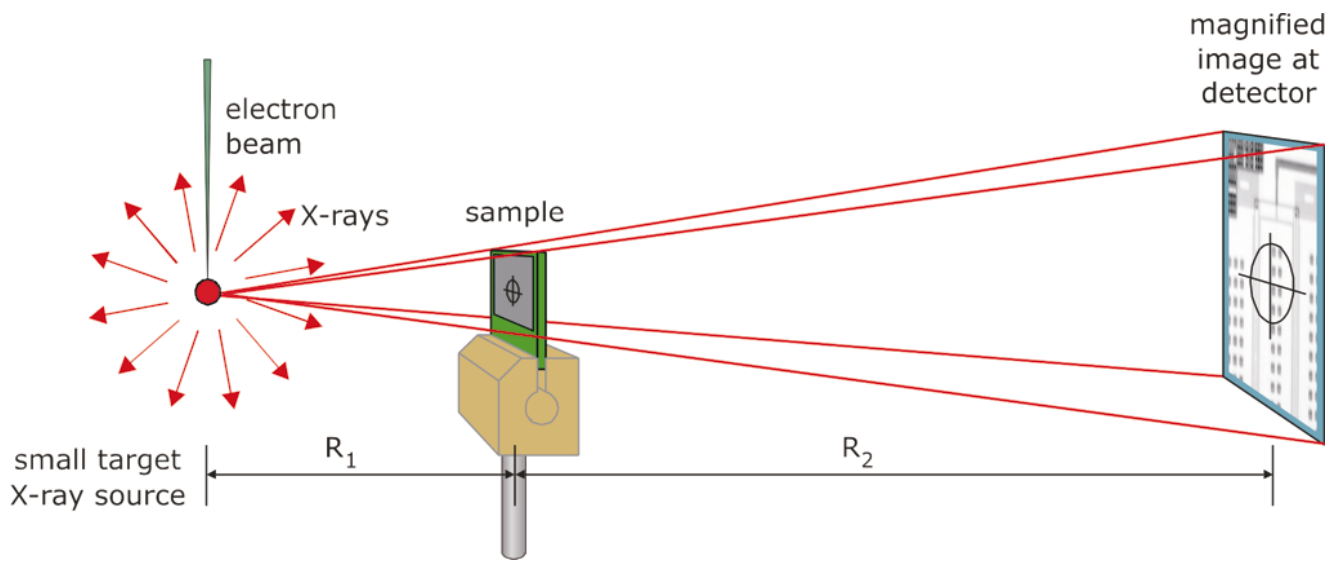

FIG. 1. Geometry of the X-ray projection microscope. Magnification $=\left(R_{1}+R_{2}\right) / R_{1}$.

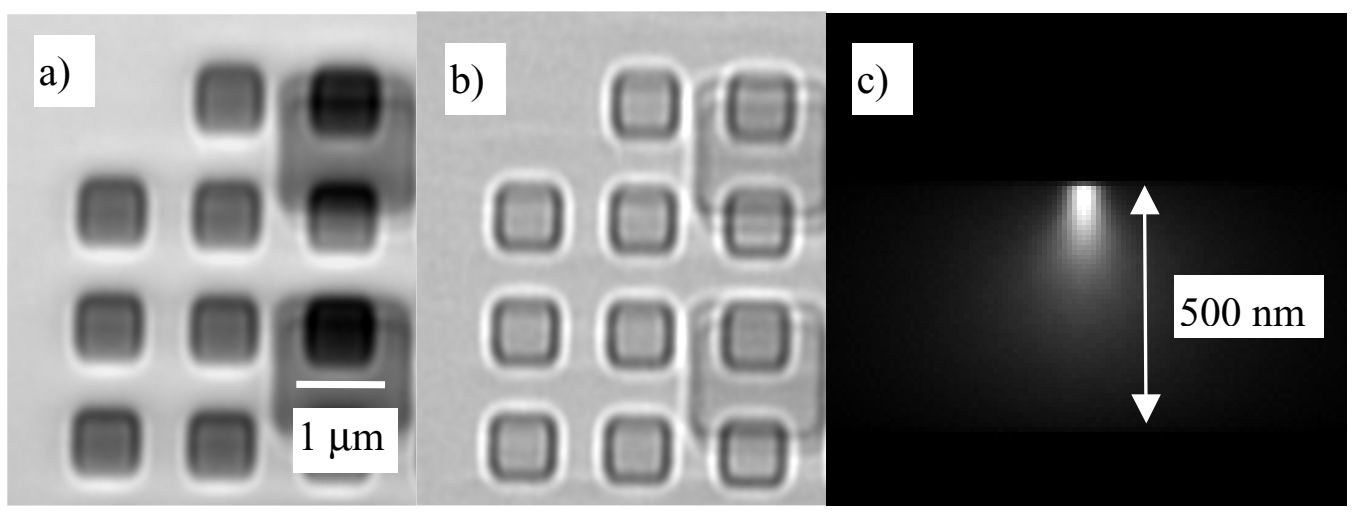

FIG. 2a Original image, FIG. 2b after deconvolution using PSF shown in FIG. 2c. 